\title{
An Integrated Program to Simulate the Multibody Dynamics of Flapping Flight
}

\author{
Anh Tuan Nguyen ${ }^{1 *}$, Thanh-Dong Pham ${ }^{1}$, Cong-Truong Dinh', Jae-Hung Han \\ ${ }^{1}$ Le Quy Don Technical University, Hanoi, Vietnam \\ ${ }^{2}$ Hanoi University of Science and Technology, Hanoi, Vietnam \\ ${ }^{3}$ Korea Advanced Institute of Science and Technology, Republic of Korea \\ "Email: anhtuannguyen2410@gmail.com
}

\begin{abstract}
This paper presents an in-house developed program that couples multibody dynamics and aerodynamics codes to simulate flapping flight of insects and micro air vehicles. The multibody dynamics code is built based on the numerical solution of the Lagrange equation, while the extended unsteady vortex-lattice method is employed to develop the aerodynamics code. The solution from the governing equation is obtained by the use of the fourth-order Runge-Kutta method and validated against the simulation results from a commercial software MSC Adams for a micro air vehicle model. In this work, parallel computing techniques are applied while estimating the aerodynamics force to minimize the running time of the program.
\end{abstract}

Keywords: Flapping flight, multibody dynamics, micro air vehicles, motion-aerodynamics interaction.

\section{Introduction}

The flapping flight of animals in the wild has been optimized by natural selection, which has lasted for hundreds of millions of years (Ellington, 1991). Nowadays, scientists have studied and adopted some important features of insect and bird flight to improve the performance of flapping-wing micro-air vehicles (FWMAVs) (Keennon and Klingebiel, 2012). In fact, flapping flight has some characteristics that do not exist in conventional fixed-wing and rotary-wing flights. While animals and micro-air vehicles flap their wings, the surrounding fluid moves in a such complex way that lift is generated to maintain a balanced flight. Moreover, the dynamics of flapping flight is characterised by the multi-degree-of-freedom motion, including six degrees of freedom (DOFs) for the body, and three DOFs for each wing. The interaction of aerodynamics and motion in flapping flight causes lots of trouble to model it. Some attempts have been made to simulate the multibody dynamics of flapping flight while considering the two-way interaction between aerodynamics and motion. Zhang and Sun (2010) coupled the NavierStokes equations and the equations of motion for insect flight. However, this type of approach suffers from an excessive computation cost due to the use of a high-order aerodynamic model. Moreover, the solutions from this simulation program have not been rigorously validated. Another approach, which ignores the contribution of wing inertia, has been widely used to simulate and study flapping flight [2]. Using this approach, the dynamics properties of

ISSN: 2734-9373

https://doi.org/10.51316/jst.150.ssad.2021.31.1.10

Received: 22 February 2020; accepted: 12 March 2021 flying systems are simplified as only the six DOFs of the body are considered. Some authors have indicated that this simplification technique is valid only for high-frequency flapping-wing systems with low wing mass. Due to these reasons, it is necessary to develop an efficient approach that is capable of modeling the multibody dynamics of flapping flight and considering the interaction between aerodynamics and motion.

a)

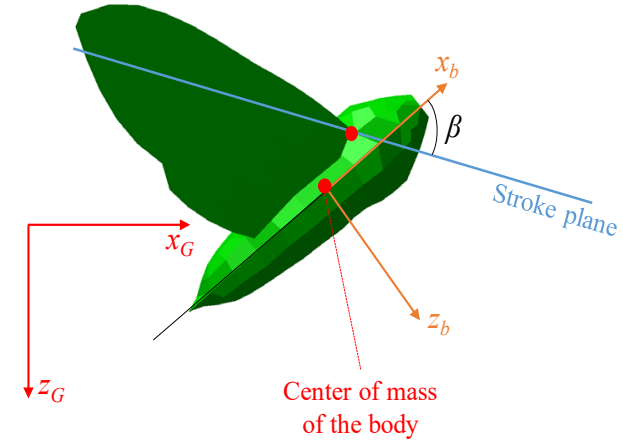

b)

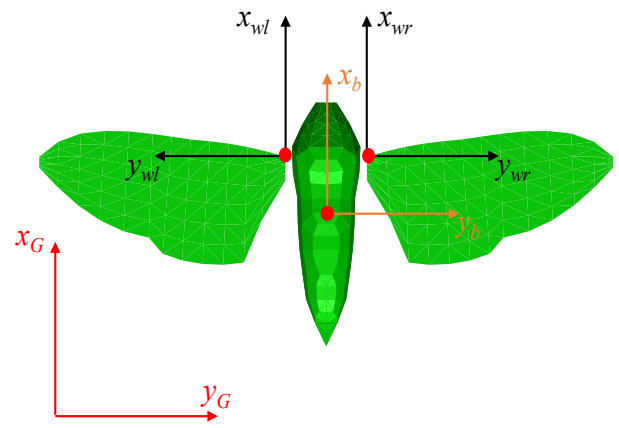

Fig. 1. The FWMAV model and the coordinate systems used in this study 


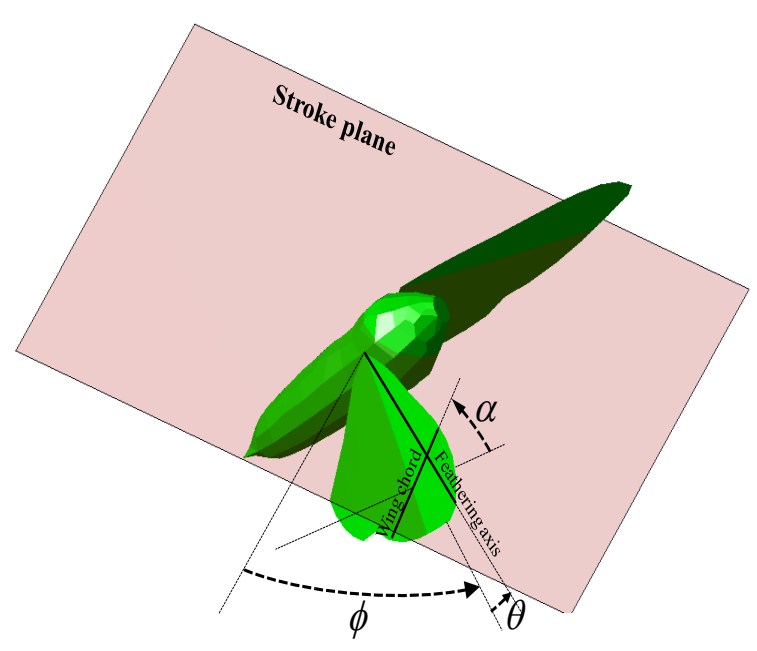

Fig. 2. Euler angles to define the wing orientation.

The program developed in this paper couples the extended unsteady vortex-lattice method (UVLM), which is regarded as an intermediate cost and fidelity aerodynamic method and the multibody dynamics solver. The validity of the multibody dynamics solver is tested in a comparison with the solution from the MSC Adams software. In this work, the program is applied to an insect-like FWMAV in hover.

\section{Methodlogy}

\subsection{FWMAV Model}

The FWMAV model used in this study has the same mass and geometric parameters as the hawkmoth Manduca sexta. The body and each wing weigh 1485.0 and $46.87 \mathrm{mg}$, respectively; the wing length is $48.5 \mathrm{~mm}$. Here, we use four coordinate systems, including the ground-fixed, the body-fixed and the two wing-fixed coordinated systems as shown in Fig. 1. In this figure, $\beta$ is an angle between the stroke plane of the wings and the body axis.

The wing orientation relative to the stroke plane is defined by three Euler angles $\phi, \theta$ and $\alpha$ (Fig. 2). The sweep angle $\phi$ defines the up and down motions of the wings, the elevation angle $\theta$ is used to control the for- and backward motions, and $\alpha$ is the rotation angle of the wings about their feathering axes. The more detailed definitions of these angles can be found in the literature [6]. The variations of $\phi, \theta$ and $\alpha$ are expressed by harmonic functions as follows:

$$
\begin{aligned}
& \phi=-\phi_{0} \cos (2 \pi f t) \\
& \theta=\theta_{0} \cos (4 \pi f t) \\
& \alpha=\frac{\pi}{2}-\alpha_{0} \sin (2 \pi f t)
\end{aligned}
$$

where $\phi_{0}, \theta_{0}$ and $\alpha_{0}$ denote the amplitudes of the sweep, elevation and rotation angles, $f$ is the flapping frequency. In this study, it is assumed that angle $\beta$ is held constant.

\subsection{Aerodynamic Model}

The aerodynamic model is based on the extended UVLM [6], which was developed using the potential flow theory [3]. The present aerodynamic model has intermediate cost and fidelity and is capable of handling the nonlinearity of the flow. Some important effects of flapping-wing aerodynamics, such as the wake capture and the leading-edge vorstex can be modeled in this work. To reduce the running time of the program while predicting the aerodynamic force, parallel computing techniques are applied. The validity of the present aerodynamic model for flapping-wing aerodynamics has been confirmed in previous studies [5].

\subsection{Multibody Dynamics Model}

The present FWMAV consists of three objects, including the body and the two wings with 12 DOFs in total. The dynamics of the FWMAV is modeled using the Lagrange method. The kinetic energy of the body $T_{b}$ and each wing $T_{w}$ are expressed as

$$
\begin{aligned}
& T_{b}=\frac{1}{2}\left(\mathbf{V}_{b}^{G}\right)^{\mathrm{T}} M_{b} \mathbf{V}_{b}^{G}+\frac{1}{2}\left(\boldsymbol{\Omega}_{b}^{b}\right)^{\mathrm{T}} \mathbf{I}_{b}^{b} \boldsymbol{\Omega}_{b}^{b} \\
& T_{w}=\frac{1}{2}\left(\mathbf{V}_{w}^{b}\right)^{\mathrm{T}} M_{w} \mathbf{V}_{w}^{b}+\frac{1}{2}\left(\boldsymbol{\Omega}_{w}^{b}\right)^{\mathrm{T}} \mathbf{I}_{w}^{b} \mathbf{\Omega}_{w}^{b}
\end{aligned}
$$

Here, $M$ is mass, $\mathbf{I}$ is the tensor of moment of inertia, $\mathbf{V}$ and $\boldsymbol{\Omega}$ are the velocity at the centre of mass and the angular velocity, respectively. The subscripts $b$ and $w$ denote the body and a wing, whereas the superscripts $G$ and $b$ refer to the ground-fixed and body-fixed coordinate system.

The velocity of the wing in the body-fixed coordinate system $\mathbf{V}_{w}^{b}$ is

$$
\mathbf{V}_{w}^{b}=\mathbf{B}_{G \rightarrow b} \mathbf{V}_{b}^{G}+\boldsymbol{\Omega}_{b}^{b} \times \mathbf{r}_{w / b}^{b}+\mathbf{V}_{w / b}^{b}
$$

where $\mathbf{B}_{G \rightarrow b}$ is the rotation matrix that transforms the velocity in the ground-fixed to the body-fixed coordinate system; $\mathbf{V}_{w / b}$ and $\mathbf{r}_{w / b}$ are the velocity and the position vector of the wing mass centre relative to the body mass centre, respectively.

The Lagrange equation for the present FWMAV can be written in the following form [8]:

$$
\begin{aligned}
& \frac{d}{d t}\left(\frac{\partial T}{\partial \dot{\eta}}\right)-\frac{\partial T}{\partial \eta}=Q_{\eta} \\
& \eta=x, y, z, \Phi, \Theta, \Psi
\end{aligned}
$$

where $T$ is the total kinetic energy; $Q$ is the generalized force derived from the virtual work done by external forces, including gravitational and aerodynamic forces; $x, y$ and $z$ are the displacements of the body centre of mass along the corresponding axes in the ground-fixed coordinate system; $\Phi, \Theta$ and $\Psi$ are respectively the roll, pitch and yaw angles that are used to define the orientation of the body according to the 3 2-1 sequence of rotations. 
The governing equation of the current multibody dynamics problem is formulated as

$$
\mathbf{M}(\Phi, t) \ddot{\boldsymbol{\Phi}}+\mathbf{H}(\Phi, \dot{\Phi}, t)=\mathbf{Q}(\Phi, t, \ldots)
$$
follows:

$\mathbf{M}, \mathbf{H}$, and $\mathbf{Q}$ matrices are given in detail as

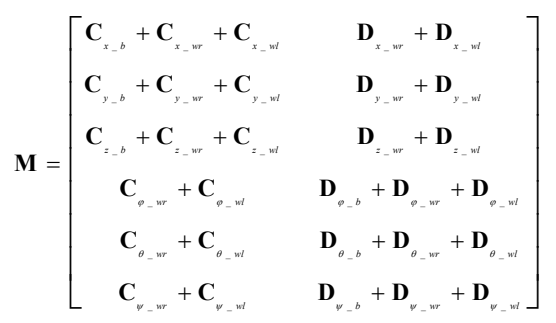

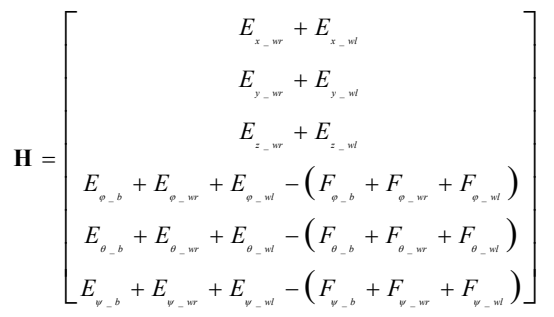$$
\mathbf{Q}=\left[\begin{array}{c}
\mathbf{F}^{\sigma} \\
\mathbf{B}_{1}^{\top} \mathbf{B}_{\sigma \rightarrow b} \mathbf{M}^{\sigma}
\end{array}\right]
$$

$\mathbf{M}, \mathbf{H}$ and $\mathbf{Q}$ matrices are obtained from equation (4). Equation (5) is solved by the fourthorder Runge-Kutta method.

The coefficients related to the body are expressed as

$$
\begin{aligned}
& \mathbf{C}_{\eta_{-} b}=M_{b} \frac{\partial \dot{\boldsymbol{\Phi}}_{1}^{\mathrm{T}}}{\partial \dot{\eta}} \\
& \mathbf{D}_{\eta_{-} b}=\frac{\partial \dot{\boldsymbol{\Phi}}_{2}^{\mathrm{T}}}{\partial \dot{\eta}} \mathbf{B}_{1}^{\mathrm{T}} \mathbf{I}_{b}^{b} \mathbf{B}_{1} \\
& E_{\eta_{-} b}=\frac{\partial \dot{\boldsymbol{\Phi}}_{2}^{\mathrm{T}}}{\partial \dot{\eta}} \frac{d\left(\mathbf{B}_{1}^{\mathrm{T}} \mathbf{I}_{b}^{b} \mathbf{B}_{1}\right)}{d t} \dot{\boldsymbol{\Phi}}_{2}
\end{aligned}
$$

Here $\eta=x, y, z, \varphi, \theta, \psi ; \mathbf{M}$ and $\mathbf{I}$ respectively denote the mass and the moment of inertia. For the body, the moment of inertia is determined about its center of mass. $\mathbf{B}_{1}$ is the matrix that converts the derivatives of the three Euler angles $\varphi, \theta, \psi$ to the angular velocity in the body-fixed coordinate system, and $\mathbf{B}$ is a transform matrix. The superscripts $b$ and $G$ refer to the body-fixed and ground-fixed coordinate systems, respectively. $\boldsymbol{\Phi}_{1}$ is $(x, y, z)^{T}$ and $\boldsymbol{\Phi}_{2}$ is $(\varphi, \theta, \psi)^{T}$.

The coefficients related to the wings are given in the following equations. $[\mathbf{r}]_{\times}$denotes the skewsymmetric matrix of $\mathbf{r}$, which is used to represent a cross product in a matrix form.

$$
\begin{aligned}
& \mathbf{C}_{\eta_{-} w}=M_{w} \frac{\partial \dot{\Phi}_{1}^{\mathrm{T}}}{\partial \dot{\eta}} \\
& \mathbf{D}_{\eta_{-} w}=M_{w} \frac{\partial \dot{\boldsymbol{\Phi}}_{1}^{\mathrm{T}}}{\partial \dot{\eta}} \mathbf{B}_{G \rightarrow b}{ }^{\mathrm{T}}\left[\mathbf{r}_{w c / b}^{b}\right]^{\mathrm{T}} \mathbf{B}_{1}
\end{aligned}
$$

$$
\begin{gathered}
E_{\eta_{-} w}=M_{w} \frac{\partial \dot{\Phi}_{1}^{\mathrm{T}}}{\partial \dot{\eta}}\left(\begin{array}{c}
\frac{d \mathbf{B}_{G \rightarrow b}^{\mathrm{T}}}{d t}\left[\mathbf{r}_{w c / b}^{b}\right]^{\mathrm{T}} \mathbf{B}_{1}+ \\
\mathbf{B}_{G \rightarrow b}{ }^{\mathrm{T}} \frac{d\left[\mathbf{r}_{w c / b}^{b}\right]^{\mathrm{T}}}{d t} \mathbf{B}_{1}+\dot{\Phi}_{2}+ \\
\mathbf{B}_{G \rightarrow b}^{\mathrm{T}}\left[\mathbf{r}_{w c / b}^{b}\right]^{\mathrm{T}} \frac{d \mathbf{B}_{1}}{d t}
\end{array}\right) \\
M_{w} \frac{\partial \dot{\Phi}_{1}^{\mathrm{T}}}{\partial \dot{\eta}}\left(\frac{\left.d \mathbf{B}_{G \rightarrow b}{ }^{\mathrm{T}} \mathbf{V}_{w c / b}^{b}+\mathbf{B}_{G \rightarrow b}{ }^{\mathrm{T}} \frac{d \mathbf{V}_{w c / b}^{b}}{d t}\right)}{d t}\right)
\end{gathered}
$$

$\eta=x, y, z$

and

$$
\begin{aligned}
& \mathbf{C}_{\eta_{-} w}=M_{w} \frac{\partial \dot{\boldsymbol{\Phi}}_{2}^{\mathrm{T}}}{\partial \dot{\eta}} \mathbf{B}_{1}^{\mathrm{T}}\left[\mathbf{r}_{w c / b}^{b}\right] \mathbf{B}_{G \rightarrow b} \\
& \mathbf{D}_{\eta_{-} w}=\left(\begin{array}{l}
M_{w} \frac{\partial \dot{\boldsymbol{\Phi}}_{2}^{\mathrm{T}}}{\partial \dot{\eta}} \mathbf{B}_{1}^{\mathrm{T}}\left[\mathbf{r}_{w c / b}^{b}\right]\left[\mathbf{r}_{w c / b}^{b}\right]^{\mathrm{T}} \mathbf{B}_{1}+ \\
\frac{\partial \dot{\boldsymbol{\Phi}}_{2}^{\mathrm{T}}}{\partial \dot{\eta}} \mathbf{B}_{1}^{\mathrm{T}} \mathbf{I}_{w}^{b} \mathbf{B}_{1}
\end{array}\right)
\end{aligned}
$$$$
E_{\eta_{-w}}=M_{w} \frac{\partial \dot{\boldsymbol{\Phi}}_{2}^{\mathrm{T}}}{\partial \dot{\eta}}\left(\begin{array}{l}
\frac{d \mathbf{B}_{1}^{\mathrm{T}}}{d t}\left[\mathbf{r}_{w e c h b}^{b}\right]_{\times} \mathbf{B}_{G \rightarrow b}+ \\
\mathbf{B}_{1}^{\mathrm{T}} \frac{d\left[\mathbf{r}_{w c l b}^{b}\right]_{\times}}{d t} \mathbf{B}_{G \rightarrow b}+ \\
\mathbf{B}_{1}^{\mathrm{T}}\left[\mathbf{r}_{w c i b}^{b}\right]_{\times} \frac{d \mathbf{B}_{G \rightarrow b}}{d t}
\end{array}\right) \dot{\boldsymbol{\Phi}}_{1}+
$$

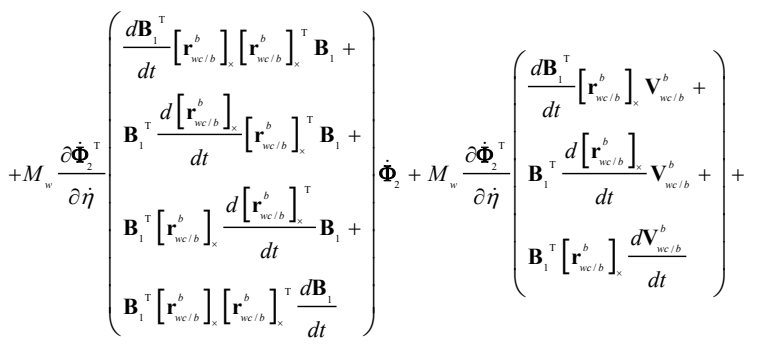$$
+\frac{\partial \dot{\boldsymbol{\Phi}}_{2}{ }^{\mathrm{T}}}{\partial \dot{\eta}}\left(\begin{array}{c}
\frac{d \mathbf{B}_{1}{ }^{\mathrm{T}}}{d t} \mathbf{I}_{w}^{b} \mathbf{B}_{1}+ \\
\mathbf{B}_{1}^{\mathrm{T}} \frac{d \mathbf{I}_{w}^{b}}{d t} \mathbf{B}_{1}+ \\
\mathbf{B}_{1}^{\mathrm{T}} \mathbf{I}_{w}^{b} \frac{d \mathbf{B}_{1}}{d t}
\end{array}\right) \dot{\boldsymbol{\Phi}}_{2}+\frac{\partial \dot{\boldsymbol{\Phi}}_{2}^{\mathrm{T}}}{\partial \dot{\eta}}\left(\begin{array}{c}
\frac{d \mathbf{B}_{1}^{\mathrm{T}}}{d t} \mathbf{I}_{w}^{b} \boldsymbol{\Omega}_{w / b}^{b}+ \\
\mathbf{B}_{1}^{\mathrm{T}} \frac{d \mathbf{I}_{w}^{b}}{d t} \boldsymbol{\Omega}_{w / b}^{b} \\
+\mathbf{B}_{1}{ }^{\mathrm{T}} \mathbf{I}_{w}^{b} \frac{d \boldsymbol{\Omega}_{w / b}^{b}}{d t}
\end{array}\right)
$$

$$
\eta=\varphi, \theta, \psi
$$

The generalized coordinate vector $\boldsymbol{\Phi}$ is

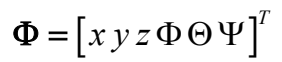

The validity of the multibody dynamics solver is examined in a comparison with the solution from the 
MSC Adams software for the same FWMAV. Here, arbitrary external forces are applied to the two wings and the body. Moreover, there is a phase difference between the motions of the left and right wings. Fig. 3 shows the displacements and the orientations of the body from the present program and from MSC Adams.

MSC Adams is multibody dynamics software, which can provide a simulation framework for flapping-wing flight. However, the integration of unsteady aerodynamics solvers into the software framework is challenging because of its large computational cost. Nguyen et al. [8] have made an attempt to do this kind of integration. However, it should be noted that in that study, the aerodynamics solver is not entirely coupled with the iterations of the dynamics solver. Instead, the aerodynamic force is calculated for each time step, and this force is assumed to be constant during the iterations of the dynamics solver. This study presents the first attempt to entirely couple the unsteady aerodynamics solver with a multibody dynamics code.

MSC Adams uses the GSTIFF solver for the multibody dynamics simulation. A predictor-corrector method is applied to obtain the solution to the dynamics equations of the system.

Fig. 5 and Fig. 6 show the comparisons for data on the right and left wings, respectively.

\section{Simulation results}

The present program is applied to the FWMAV in hover. The wing kinematic parameters are similar to those of a real hawkmoth (Willmott and Ellington, 1997) (Table 1). The initial velocities and the angular velocities are 0 , and the initial pitch angle is $39.8 \mathrm{deg}$.

Running the simulation with the present program for the hovering FWMAV we can obtain the flight trajectory. Next, constant lateral and vertically downward gust disturbances with the magnitude of $1.0 \mathrm{~m} / \mathrm{s}$ is applied to the FWMAV, and the responses of the FWMAV to these disturbances are investigated.

Table 1. Wing kinematic parameters

$\begin{array}{cc}\text { Parameters } & \text { Values } \\ \phi_{0}(\mathrm{deg}) & 60.0 \\ \theta_{0}(\mathrm{deg}) & 10.0 \\ \alpha_{0}(\mathrm{deg}) & 57.3 \\ f(\mathrm{~Hz}) & 26.1 \\ \beta(\mathrm{deg}) & 54.8\end{array}$

The variations of the trajectory parameters within the time course of one wingbeat stroke cycle corresponding to the cases with and without the gust disturbances are shown in Fig. 6 and 7.
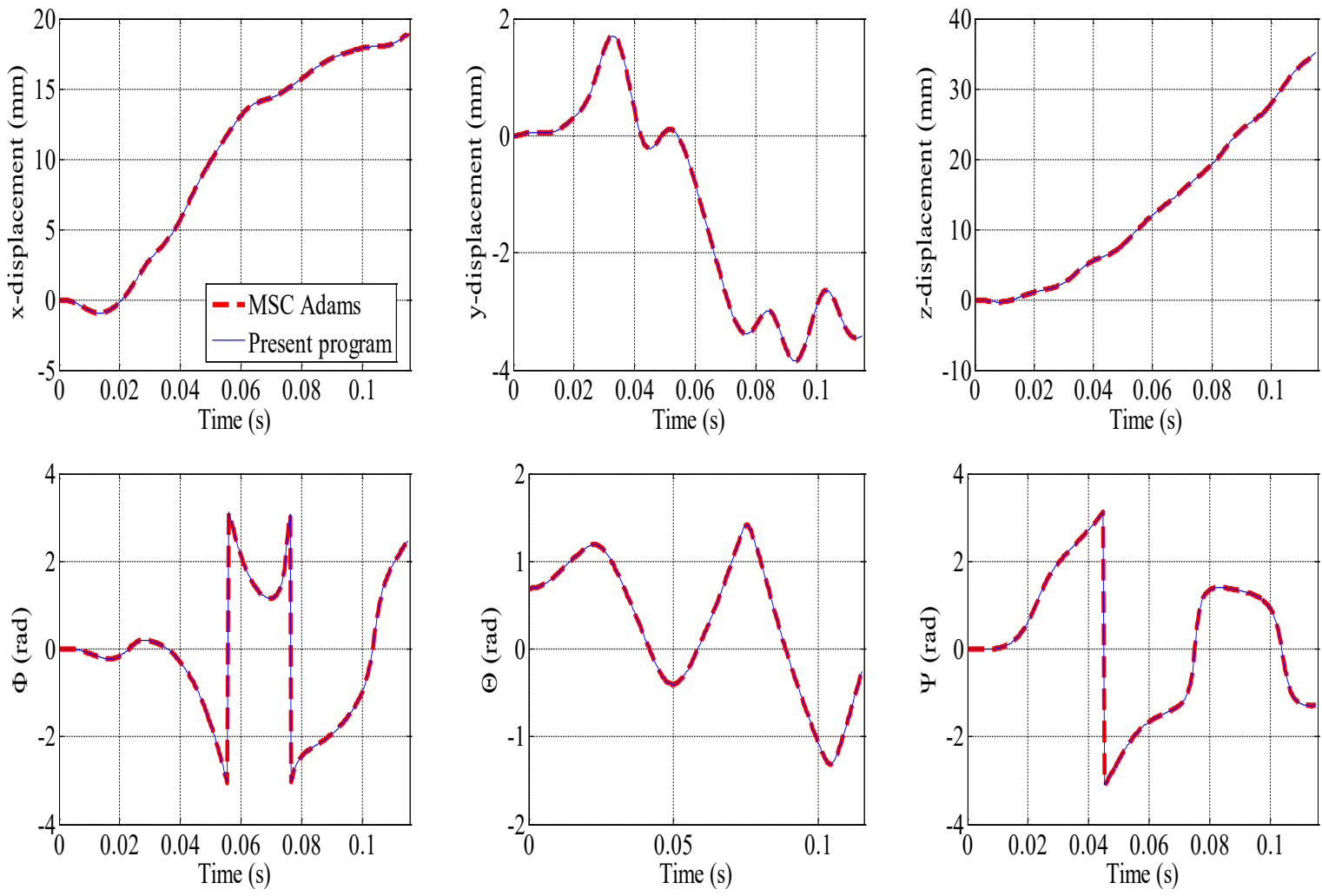

Fig. 3. The validation against the MSC Adams software for the displacements and orientations of the body 

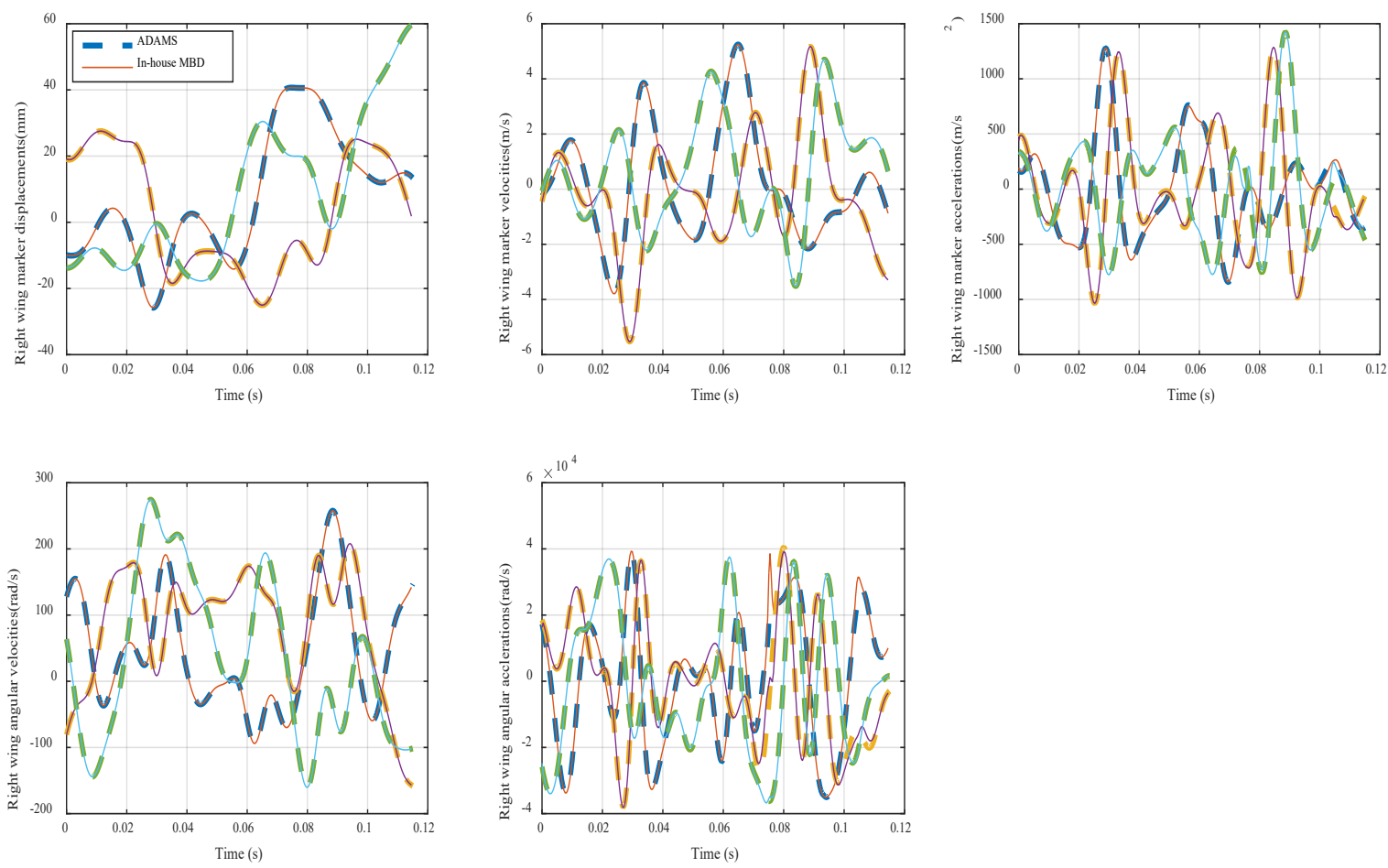

Fig. 4. The validation against the MSC Adams software for the displacements of a marker on the right wing and the angular velocity and acceleration of right wing about three axes of the ground-fixed coordinate system
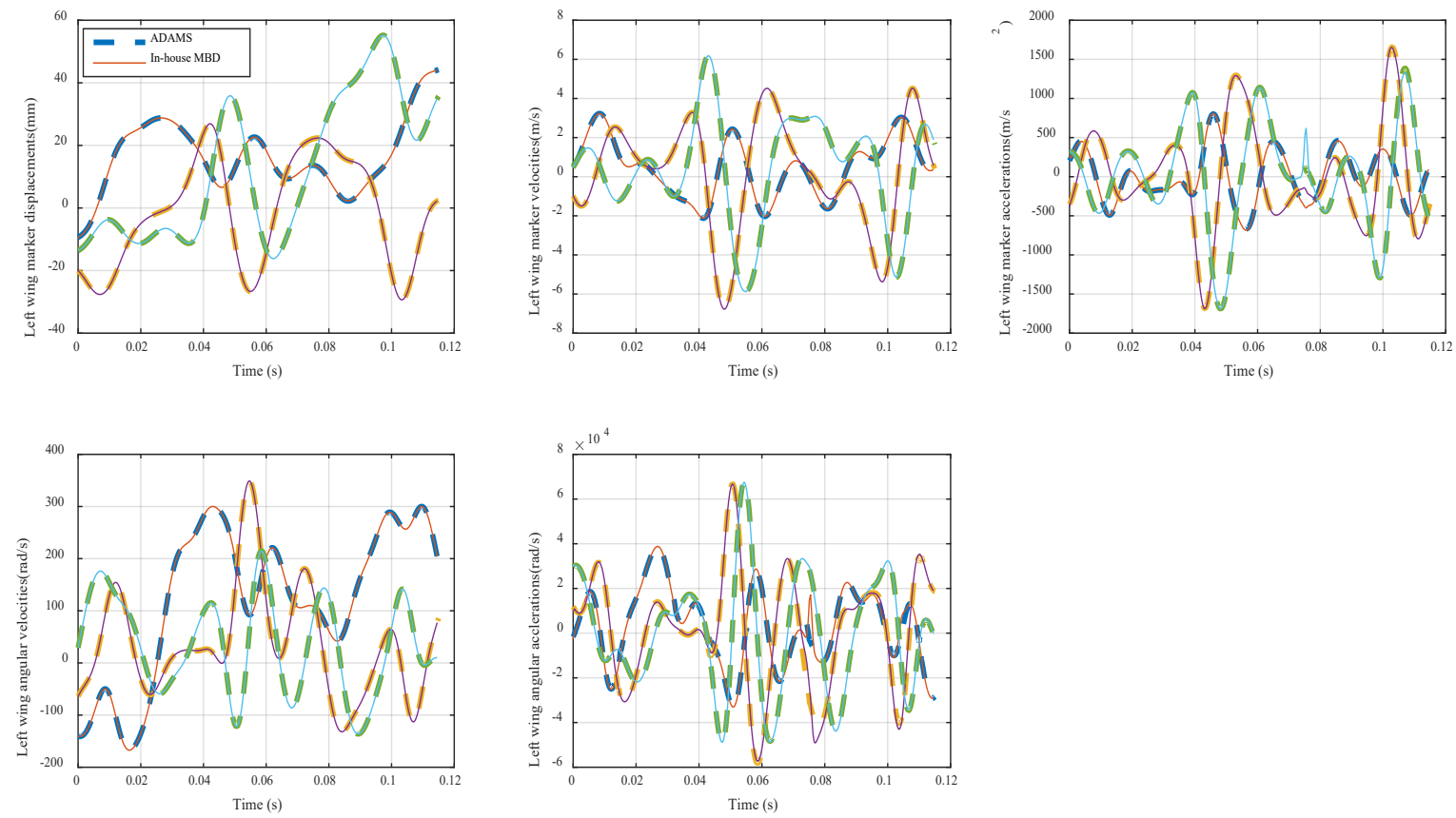

Fig. 5. The validation against the MSC Adams software for the displacements of a marker on the left wing and the angular velocity and acceleration of left wing about three axes of the ground-fixed coordinate system 

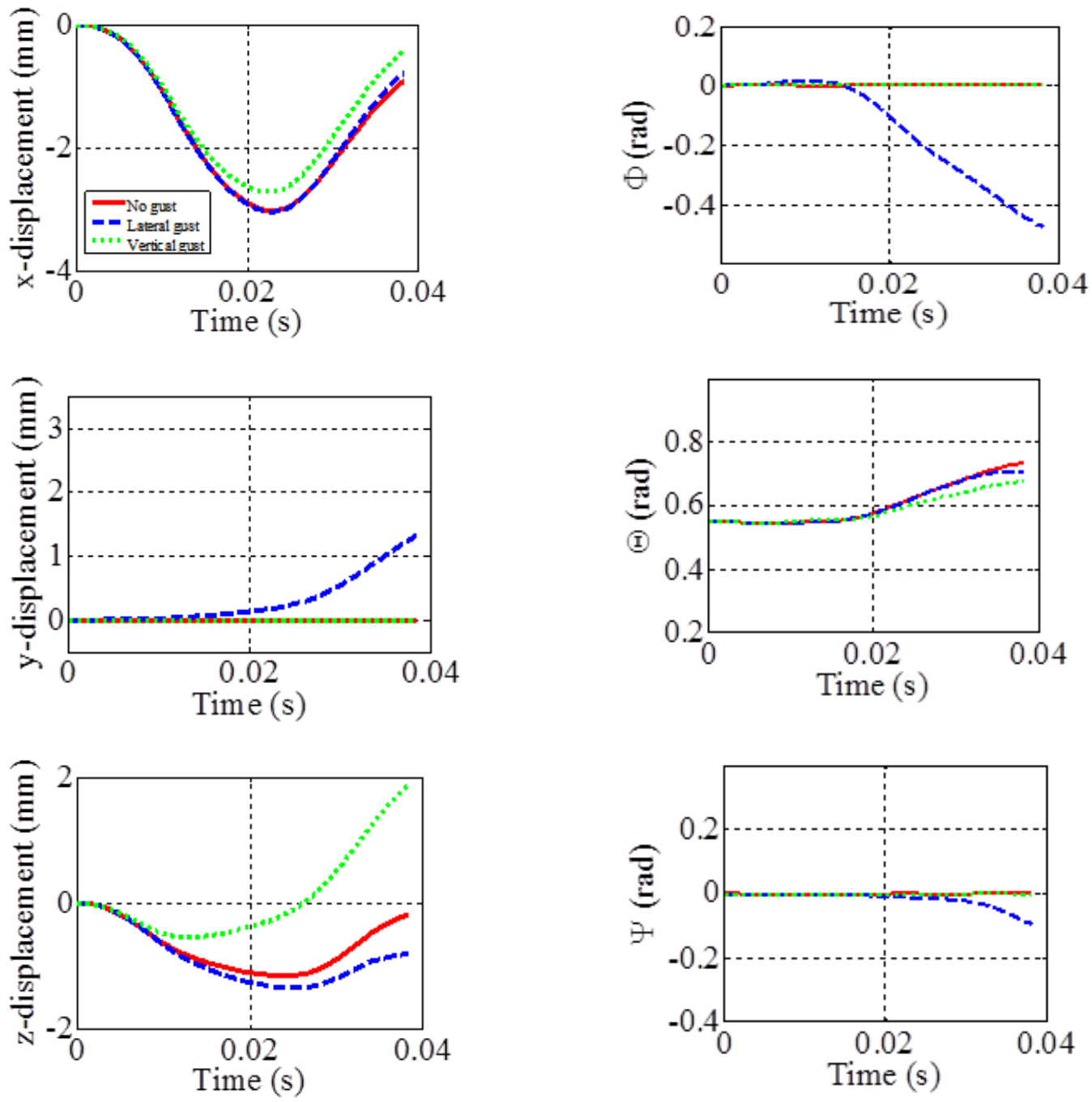

Fig. 6. Displacements in the cases of lateral, vertical Fig. 7. Euler angles of the body in the cases of lateral, and no gust disturbances vertical and no gust disturbances

a)
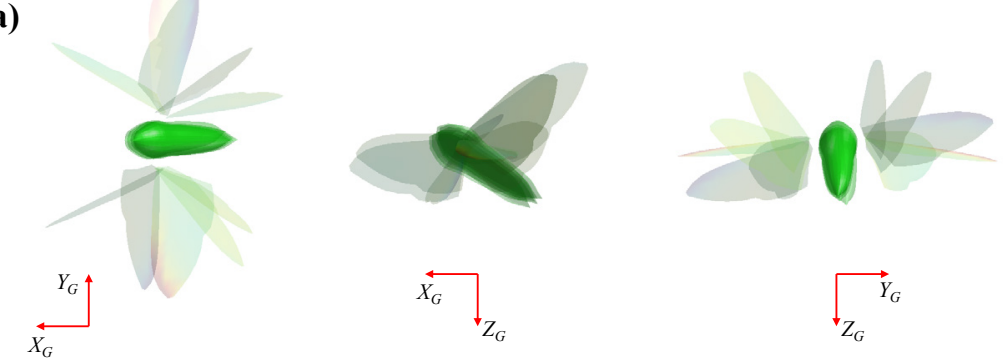

b)
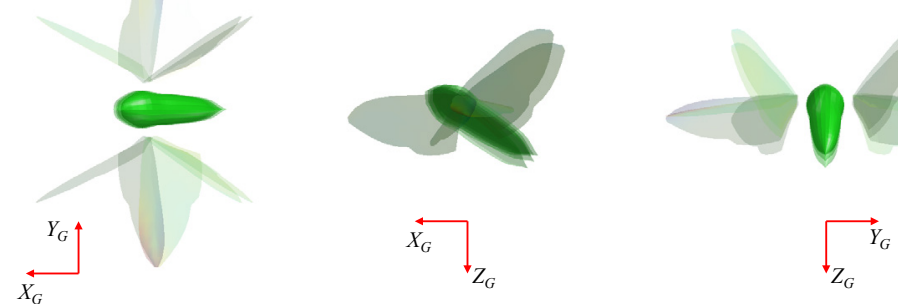

Fig. 8. The movements of the body and the wings in the cases of the lateral gust (a) and the vertically downward gust (b) 


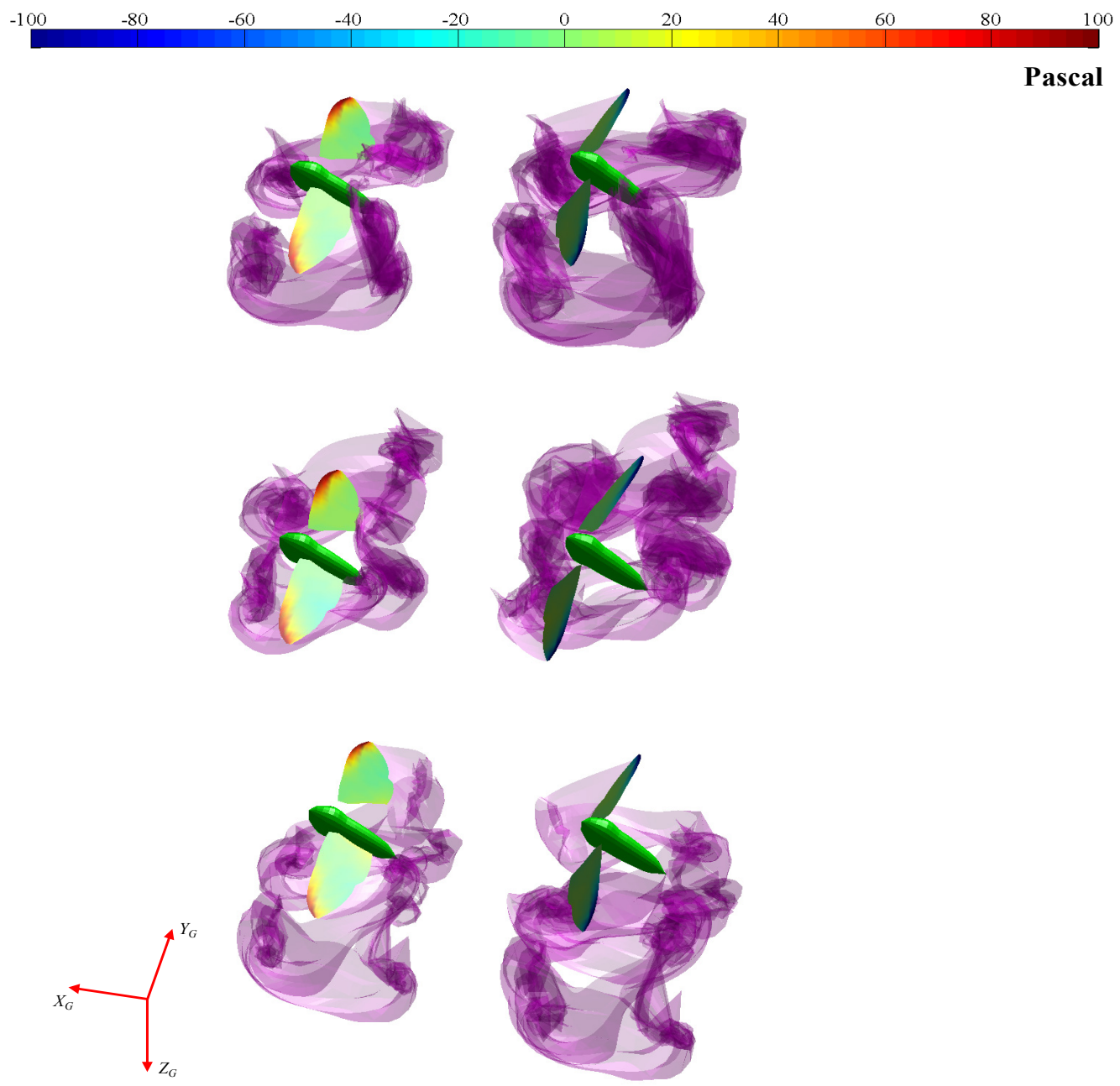

Fig. 9. Wakes and pressure difference in the case of no gust (up), lateral gust (middle) and downward gust (down). The left subfigures show the simulation in the mid of the downstroke, the right ones are corresponding to the mid of the upstroke

It is seen that when the lateral gust is applied, the FWMAV is deflected largely about the roll axis and the motion along the $y$ axis is divergent. Similarly, in the case of the vertically downward gust, the pitch angle decreases and the FWMAV moves downwards.

From these data, it is possible to state that in hovering flight, the FWMAV is dynamically unstable. The movements of the body and the wings of the FWMAV in the cases of the gust disturbances are illustrated in Fig. 8. It is seen that the lateral gust disturbance causes a bank turn motion. On the other hand, the insect slightly pitches down in the case of the downward vertical gust disturbance. This response has demonstrated the dynamic instability of the system.

Fig. 9 shows the wakes and nondimensional pressure difference on the wings in the mid of the downstroke and upstroke phases. Here, data are nondimensionalized by the reference dynamic pressure, in which we use the mean wing-tip speed. It is seen that the wake moves with the gust direction.
Moreover, strong wing-wake interaction can be visualized in this figure, which is the source of the challenge while handling the coupling between the unsteady aerodynamics solver and the multibody dynamics code.

\section{Conclusions}

The paper has presented an in-house program developed for the simulation of flapping flight. The motion-aerodynamics interaction problem is handled by the integration of the multibody dynamics and aerodynamic codes. The multibody dynamics solver was validated against the MSC Adams software. When applying the program to a hawkmoth-like flapping-wing micro air vehicle model, the results showed that this air vehicle is dynamically unstable and easily deflected due to gust disturbances.

\section{Acknowledgments}

This research is funded by Vietnam National Foundation for Science and Technology Development (NAFOSTED) under grant number 107.01-2018.05. 


\section{References}

[1] Ellington, C. P. (1991). Aerodynamics and the Origin of Insect Flight. Advances in Insect Physiology, 23, pp. 171-210.

https://doi.org/10.1016/S0065-2806(08)60094-6

[2] Lee, J.-S., Kim J.-K., and Han J.-H. (2015). Stroke Plane Control for Longitudinal Stabilization of Hovering Flapping Wing Air Vehicles. Journal of Guidance, Control, and Dynamics, 38(4), pp. 800805.

https://doi.org/10.2514/1.G000599

[3] Katz, J., and Plotkin, A. (2001). Low-Speed Aerodynamics: From Wing Theory to Panel Methods. Cambridge University Press, New York. https://doi.org/10.1017/CBO9780511810329

[4] Keennon, M., and Klingebiel, K. (2012). Development of the nano hummingbird: A tailless flapping wing micro air vehicle. In 50th AIAA Aerospace Sciences Meeting Including the New Horizons Forum and Aerospace Exposition, p. 588. https://doi.org/10.2514/6.2012-588

[5] Nguyen, A. T., Han, J. S., and Han, J. H. (2016a). Effect of body aerodynamics on the dynamic flight stability of the hawkmoth Manduca sexta. Bioinspiration \& Biomimetics, 12(1), p. 016007. https://doi.org/10.1088/1748-3190/12/1/016007

[6] Nguyen, A. T., Kim, J. K., Han, J. S., and Han, J. H. (2016b). Extended unsteady vortex-lattice method for insect flapping wings. Journal of Aircraft, 53(6), pp. 1709-1718. https://doi.org/10.2514/1.C033456

[7] Willmott, A. P., and Ellington, C. P. (1997). The mechanics of flight in the hawkmoth Manduca sexta. I. Kinematics of hovering and forward flight. Journal of experimental Biology, 200(21), pp. 2705-2722. https://doi.org/10.1242/jeb.200.21.2705

[8] Wittenburg, J., (2008). Dynamics of multibody systems. Springer, Germany.

[9] Zhang, Y. L., and Sun, M. (2010). Dynamic flight stability of hovering model insects: Theory versus simulation using equations of motion coupled with Navier-Stokes equations. Acta Mechanica Sinica, 26(4), pp. 509-520. https://doi.org/10.1007/s10409-010-0360-5 